MATEC Web of Conferences 31, 16003 (2015)

DOI: $10.1051 /$ matecconf/ 20153116003

(C) Owned by the authors, published by EDP Sciences, 2015

\title{
Research of Statistical Method for the Number of Leaves in Plant Growth Cabinet
}

\author{
Danni $\mathrm{Ci}^{1, a}$, Shigang Cui ${ }^{1}$, Fan Liang ${ }^{1}$ \\ 1 Tianjin University of Technology and Education, Tianjin ,China
}

\begin{abstract}
With the continuous decrease of arable land, the emergency of plant growth cabinet can efficiently provide people with more high quality green vegetables. The reasonable density in plant growth cabinet becomes our primary problem. The key to reasonable density is reasonable planting density, that is to say, a large coefficient of leaf area (but not too big) is needed. Vegetable leaf' quantity and the size of the leaf area can reflect whether the plants are in a good condition. It is the key to a reasonable density. But in the process of calculating vegetable leaf number, a problem is that leaves may block each other, which is not desired in subsequent statistic. Therefore this paper proposes a watershed segmentation based on improved marker control in order to get the number of connected components or the number of leaves. The experiment shows that this method can separate the overlapping leaf images effectively and thus successfully solves the target adhesion phenomenon on the subsequent analysis and measurement of interference problem.
\end{abstract}

\section{Introduction}

The agricultural development of our country is faced with a big challenge, which we must use limited resources to satisfy people's growing demand for food[1-2]. The appearance of Plant growth cabinet provides effective technical support for vertical space development of future agriculture, and provides the technical support for planting in certain areas which there are scarce land resources. The size of a leaf has a significant on the water evaporation, water balance[3-4], material cycle and economic efficiency, and also has an important influence on the crop growth and development, resistance and the final production. It is also an important index for the measure of plants' production statement and for the use of light energy utilization of plants. That planting density should

be reasonable is the key to reasonable density, that is to have a larger coefficient of leaf area,

but not too big. If the coefficient of leaf area is too large, it can make the leaf shade with each other in the plant, then the illumination of medium and bottom of plant were weaken, which result in reducing the average total leaf photosynthetic rate. Too densely planting can also lead to poor ventilation in cabinet and unfavorable to crops in carbon dioxide layer of transportation and supply, which was easy to cause the phenomenon such as undergrowth, diseases etc. The lush degree of leaves is directly related to the discretion of the light energy utilization. Within a certain range, the leaf area coefficient and light energy utilization[5] were positive. But when leaf area is too large, it can reduce the average

\footnotetext{
a Corresponding author: 15222719380@163.com
}

group total leaf photosynthetic rate. To make the group has the largest light energy utilization, we should find out the optimum range of leaf area and the best number.

In the process of detection of vegetable leaf number, we find the problem of leaf block each other, which bring a large number of difficulties to calculate the leaf number. Traditional method of identifying overlap or adhesion mainly includes the Hough transform[6], the traditional watershed algorithm, as used as the method of geometry. There are problems of recognition unaccuracy and image segmentation in the study of identifying leaf overlap or adhension. So cause some difficulties to identify the application of the algorithm. In order to improve the accurate rate of recognition of leaf overlap or adhesion, the commonly used watershed algorithm[7] was improved in this paper, and then segmentate the target image. Watershed algorithm and its improved algorithm have good capability of edge detection and accurate positioning and have good detection effect. It is a good way to solve the target adhesion phenomenon and to get the number of connected components[8], which are the leaf numbers.

\section{Materials and methods}

The growth of vegetable leaves have diversity and randomness, Sparse degree of each are not identical, this leads to the different condition of leaf block, So we have to divided into two cases to statistics on the number of leaves. One is not cover between leaves, the other exist shade or adhesion between leaves. For the second kind of complicated situation, we adopt a watershed segmenta- 
tion based on improved marker control, Firstly marked difference for the prospect of object and background objects in an image, secondly gone for distance transformation, and finally using watershed segmentation algorithm, to obtain the number of connected components or the number of leaves. The flow chart of statistics on the number of leaves of plant growth in the cabinet as shown in figure 1 below.

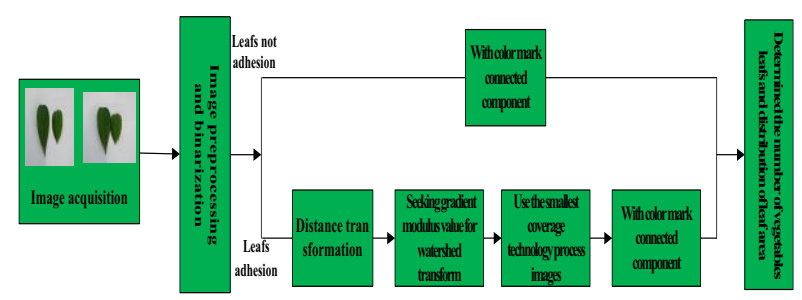

Figure1.The flow chart of statistics on the number of leaves in plant growth cabinet

\subsection{Experimental environment and Image acquisition}

This experiment under the environment of intelligent plant growth cabinet, in order to ensure various nutrient elements necessary for plant growth and to provide appropriate growth environment, the test using the method of standard hydroponic nutrient, through the intelligent control panel to adjust incase the

environment parameter, Set the LED light source to the white light source, the illumination is set to 3000LUX, the temperature is set to 23 degrees Celsius, humidity is set to $60 \%$, the concentration of carbon dioxide is set to $600 \mathrm{ppm}$.

Through the camera installed in the cabinet of the plant growth (13000000 pixels) and image acquisition device to obtain image information of the leaf, Analysis and statistics of image, the number of leaf vegetables, to analyze and count the images, the number of the vegetables leaves is obtained. The test of the vegetable leaf counting system is as the figure 2. The system includes planting vegetables growth cabinets, image acquisition system and the camera, this namely transfer the leaf information into the image information that computer can identify, Finally, the acquisition of leaf image segmentation, analysis of the connected components of vegetable leaves, the number of leaves is obtained. The environment of plant growth cabinet is shown in figure 3 as follows:

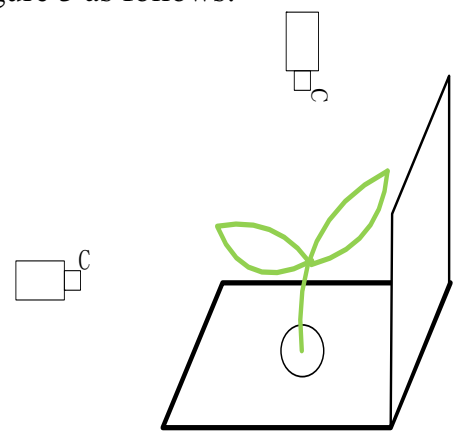

Figure 2 . Vegetable leaf counting system.

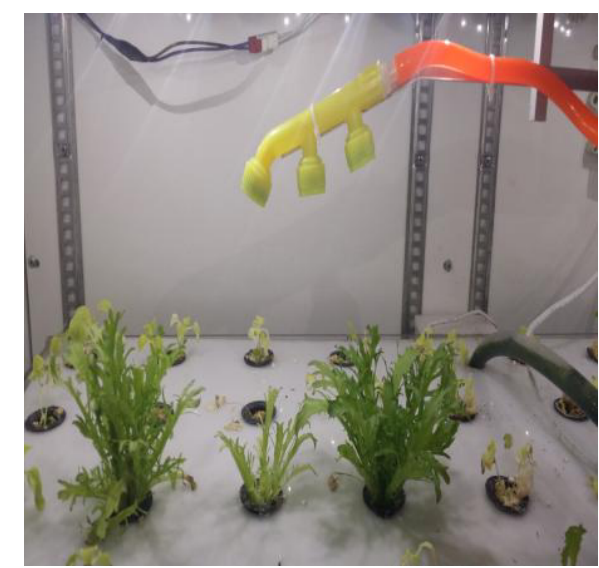

Figure 3 .The environment of plant growth cabinet.

\subsection{Image Preprocessing and Binarization}

Digital image in the process of acquisition and transmission, will be affected by a variety of noise interference and decreased image quality, So we must to smooth the image to eliminate noise and improve the image quality before image segmentation and reduce the difficulty of the subsequent handling. The betweencluster variance (Otsu) algorithm is based on one dimensional histogram of the image, in order to target and the background of maximum variance for threshold selection criterion, however, because it is based on the adaptive selection of global threshold, the image of uneven illumination can not take into account its bright and dark area, it will appear error segmentation phenomenon. The morphological opening operation has translation invariance and incremental, while ensuring no global geometric distortion. In addition, since the image in the target area and prospect is inconsistencies, partition of binary image is more or less the existence of some discrete noise, pitting on the boundary of the target area may also exist burrs or depression. Therefore, in this paper, combining the theory of morphology and Otsu threshold segmentation algorithm realize image preprocessing and binarization algorithms.

\subsection{The adhesion of leaf image segmentation}

Watershed segmentation algorithm, image segmentation is a concept with the help of topography, the algorithm can use some method of mathematical morphology. Due to its computational speed, they can pinpoint a weak edge image in the adhesion area. Watershed algorithm use image segmentation of pixel values to represent the height of that point, have great grey value of pixels corresponding to the watershed line, while with low grey value of pixels corresponding to the water basin. But the traditional watershed transform is easily affected by noise, and there is a serious phenomenon of excessive segmentation. Therefore, how to overcome the excessive segmentation is always the key point of the research of watershed algorithm. In this paper, with the tag method to overcome the watershed segmentation, after some transformation to the image as the input image of 
watershed algorithm, in the watershed algorithm for adhesion image segmentation, watershed segmentation algorithm of binary image is the object distance diagram, distance map is usually based on binary image distance transform or limit corrosion receive, in this paper using distance transformation for distance map.

\section{The result of the experiment and analysis}

\subsection{Leaf not cover}

Through the application of relevant technology of digital image processing, there will be no adhesion of the leaf with color marked, so that intuitive display, finally we can statistics on the number of leaves and distribution of leaf area. The leaf of the original image as shown in figure 4.The leaf image after color marked as shown in figure 5.As shown in figure 6 we calculated the number of connected component marked, namely the number of leaves is 2 , while the results of the distribution of the two leaves of leaf area.

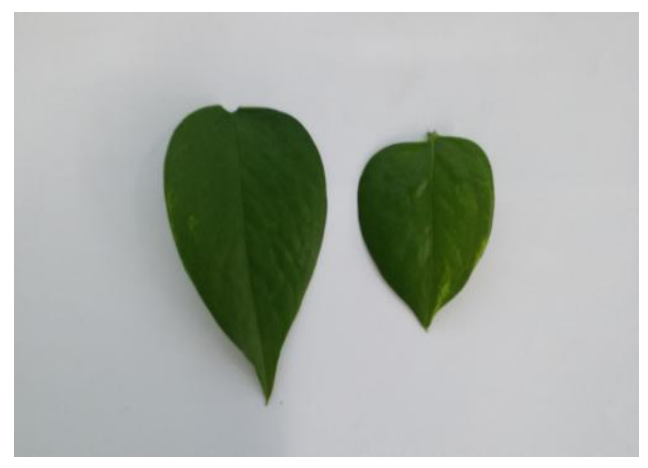

Figure 4. Leaf of the original image.

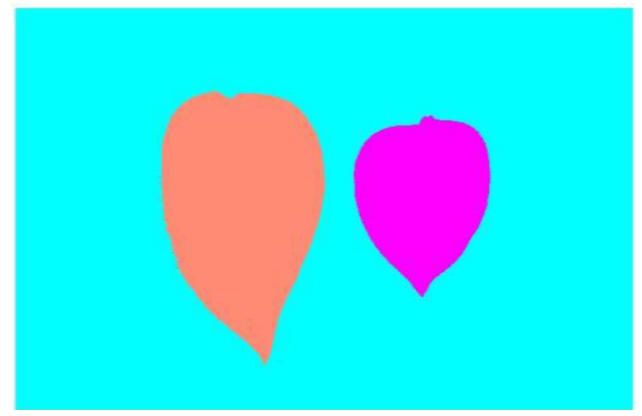

Figure 5. Leaf image after color marked.

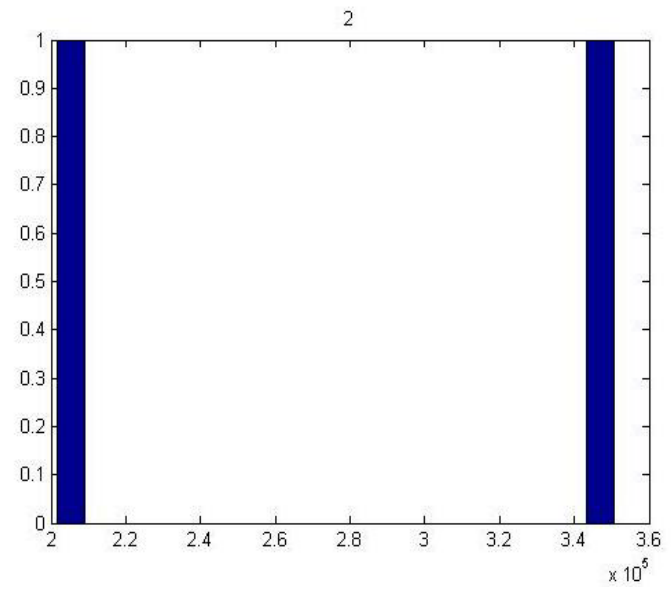

Figure 6. The results of number of leaves and distribution of leaf area.

\subsection{Leaf covered or adhesion}

For leaf image with adhesion after binarization conduct distance transformation, and the normalized to $0 \sim 1$, the distance transformation in 0.5 as a threshold for binarization, then obtaining the number of connected components is the number of leaves. Because the gradient magnitude image along the edges has bigger modulus value, therefore, ideally, the watershed transform in the gradient image can get the watershed ridge line along the edges. In this paper, sobel operator seeking gradient magnitude, then the smallest coverage technology process images, so that the local minimum area only in the marked position, carry on Watershed transformation, and finally with different colors to mark each of the leaves foreground objects and background, find the number of leaves. The original leaf image as shown in figure 7 . The leaf image after binarization and distance transform as shown in figure 8, we calculated the number of connected component labeling, namely the number of leaf is 2 . The leaf image after color marked as shown in figure 9.

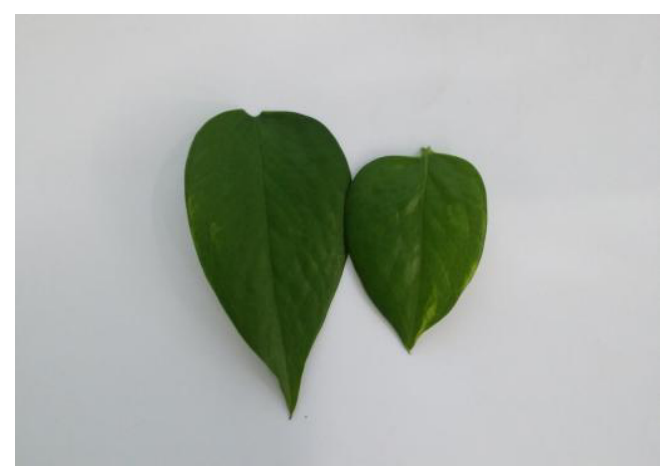

Figure 7 .The original leaf image. 


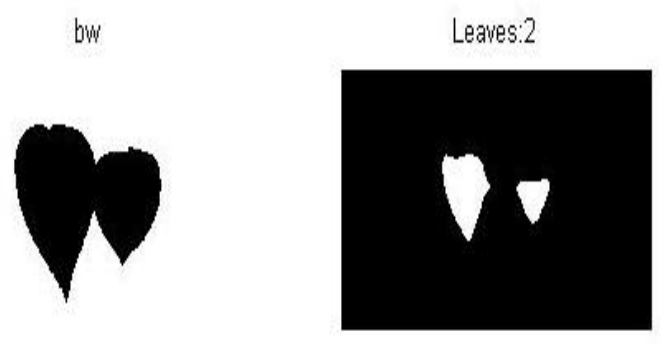

Figure 8. Leaf image after binarization and distance transform.
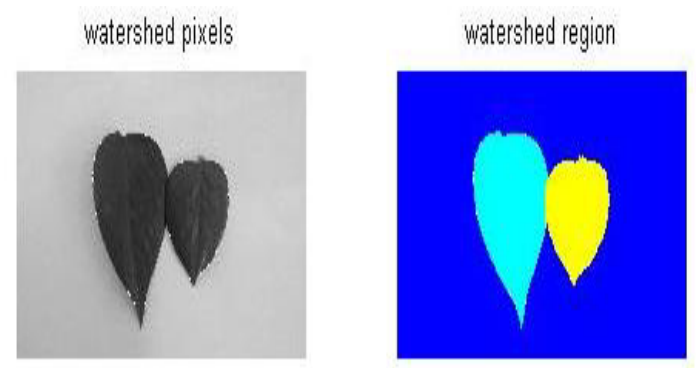

Figure 9. Leaf image after color marked.

In this study, under Windows 7 environment, using MATLAB7.1 programming to statistics the number of vegetable leaves, Regardless of whether there is shelter leaf, the leaf on the original image can be well segmented, successfully determined the number of vegetables leaves.

\section{Conclusion}

This paper, aiming at the existence of Adhesion problems with the images of the collection in the process of Statistics the number of leaves, proposing a watershed segmentation based on improved marker control. In the process of gain distance map, the structural elements used to obtain distance map, in the process of watershed segmentation, watershed segmentation of distance map, and extract the real watershed, finally get the number of connected components, you can get the number of leaves. It provides the theory basis for subsequent determine the number of leaf vegetables in plant growth cabinet, also lay the foundations for the reasonable density of plant growth cabinet. It make vegetables more efficient use of plant growth cabinet light source, and provide more high quality vegetables.

\section{References}

1. Hu Xiaohong, Li Bingjun, Xi Lei. Multi-instance graph approach to wheat leaf disease segmentation. J. Transactions of the Chinese Society of Agricultural Engineering Transactions of the CSAE,28,154 (2012).

2. WANG ping, TANG jiang-feng, WANG Bo, WANG Li-di. Image segmentation method of plant leaves based on mathematical morphology. J. Acta Agriculturae Zhejiangensis, 24,509 (2012).

3. HUANG Ling, HU Bo, CAO Nai-wen. The Novel Fries-Counting Method Based on Image Processing. J .Hubei Agricultural Sciences, 51,1880 (2012).
4. Xun Yi, Bao Guanjun, yang Qinghua, et al. Automatic segmentation of touching corn kernels in digital image. J . Transaction of the Chinese Society for Agricultural Machinery, 41,163(2010).

5. Bieniek A, Moga A.An efficient watershed algorithm base on connect components.J. Pattern Recognition, 907 916 (2000).

6. Wang Kaiyi, Zhang Shuifa, Yang Feng, et al.Online segmentation of clustering Chinese Society for Agricultural Machinery, 38,106(2007).

7. Xiang Rong, Ying Yibin, Jiang Huanyu, et al. Recognition of Overlapping Tomatoes Based on Edge Curvature Analysis. J .Transaction of the Chinese Society for Agricultural Machinery, 43, 157(2012). 